\title{
Impact of magnetic perturbation coils on the edge radial electric field and turbulence in ASDEX Upgrade
}

\author{
G.D.Conway, S.Fietz, H.W.Müller, T.Lunt, P.Simon, \\ W.Suttrop, M.Maraschek, T.Happel, E.Viezzer, and \\ ASDEX Upgrade Team
}

Max-Planck Institut für Plasmaphysik, Boltzmannstasse 2, 85748 Garching, Germany

\begin{abstract}
The impact of applied magnetic perturbations (MPs) on tokamak edge parameters has been investigated in ASDEX Upgrade low collisionallity L-mode discharges using a flexible set of in-vessel saddle coils (capable of generating $n=0,1,2$ and 4 toroidal modes) and an extensive set of high resolution edge diagnostics. Doppler reflectometry is used principally to probe the MP field penetration and structure via the radial electric field $E_{\mathrm{r}}$ and density fluctuation $\delta n_{\mathrm{e}}$ behaviour. Different MP response behaviour are observed for the near scrape-off-layer, SOL, (where $E_{\mathrm{r}}$ flattens) and in the confinement region (where the negative $E_{\mathrm{r}}$ well reverses). The radial structure of $E_{\mathrm{r}}$ and $\delta n_{\mathrm{e}}$ are particularly sensitive to the degree of MP resonance with the edge rational field-lines. Specifically, the edge turbulence is enhanced for strongly resonant MPs and reduced when non-resonant. The toroidal structure of the MP response has also been probed for various MP configurations by rotating the MP field toroidally, and is found to be different for the edge and near SOL regions.
\end{abstract}

PACS numbers: $52.25 . \mathrm{Fi}, 52.55 . \mathrm{Fa}, 52.35 . \mathrm{Ra}, 52.70 . \mathrm{Gw}$

Submitted to: Plasma Phys. Control. Fusion

Version of: 24 October 2014 


\section{Introduction}

The use of magnetic perturbation (MP) control coils for modifying the ELM and MHD behaviour in tokamak H-mode plasmas has become again an intense topic of investigation in recent years with many machines operating a variety of in- and ex-vessel saddle coils $[1,2,3,4,5]$. Although the theory and application of small perturbing magnetic fields is not new, cf. TEXT and JFT-2M results from the 1990's [6, 7, 8], the nonlinear response of the plasma to the MPs is rather complex and there remain many open questions. For example, a recent aspect is the role of the MP on the edge turbulence and zonal flows and their impact on the ELM cycle [9]. Nevertheless, to investigate the fundamental plasma response to MPs it is generally more convenient to operate in regimes where the plasma response is strongest and clearest to observe namely low density L-mode plasmas, cf. $[10,11,12,13]$. ASDEX Upgrade (AUG) is particularly well suited to this task with its flexible set of in-vessel saddle coils (detailed in section 2) which are capable of generating MPs with (predominant) toroidal mode numbers $n=0,1,2$ and 4; and its extensive set of high resolution edge diagnostics.

Langmuir probes have been widely used to measure plasma potential and density variations (which are seen to be particularly sensitive indicators of the MP effect) in the plasma scrape-off-layer (SOL) and separatrix regions of tokamaks $[12,14,15,16]$, while deeper probing has been achieved with reflectometry [17], CXRS [11, 18], BES [9] and HIBP [7] for example. In this work, Doppler reflectometry (described in section 3) is used on AUG to directly measure the radial electric field $E_{\mathrm{r}}$, its fluctuations, as well as the density turbulence $\delta n_{\mathrm{e}}$ behaviour with high spatial resolution from the far SOL to inside the edge gradient region of low collisionality L-mode discharges.

Here, new results are presented showing different MP plasma response behaviour for the open field-line SOL - where flattening of the $E_{r}$ and $n_{e}$ profiles is seen in the near SOL

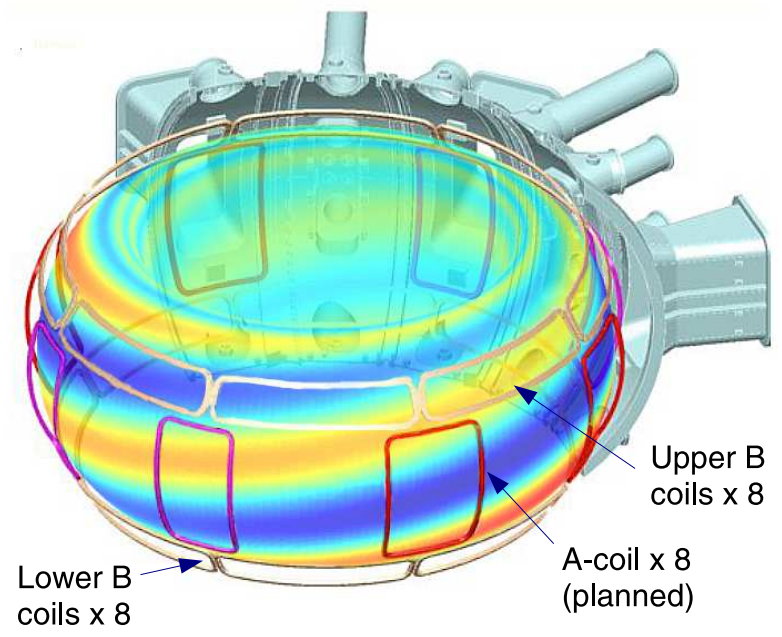

Figure 1. A 3D view of the AUG MP coils and plasma with representative perturbation field together with cut-away of the vacuum vessel. 
- and for the stochastic confinement region where the negative $E_{r}$ well reverses (sections $4 \& 5)$. The extent and radial structure of the $E_{\mathrm{r}}$ and $\delta n_{\mathrm{e}}$ are found to be particularly sensitive to the MP configuration, as well as the MP parity/phasing and the degree of MP field resonance with the edge rational field-lines. Specifically, the turbulence is locally enhanced when the MP configuration is significantly resonant (section 6). The 3D structure of the MP has also been investigated for various MP configurations by rotating the MP field toroidally. The toroidal variation of the $E_{\mathrm{r}}$ and $\delta n_{\mathrm{e}}$ radial profiles are found to be different for the edge and near SOL regions (section 7). Although the focus here is on $n=2 \mathrm{MPs}$, the overall behaviour is similar for $n=1$ and $n=4$ MPs.

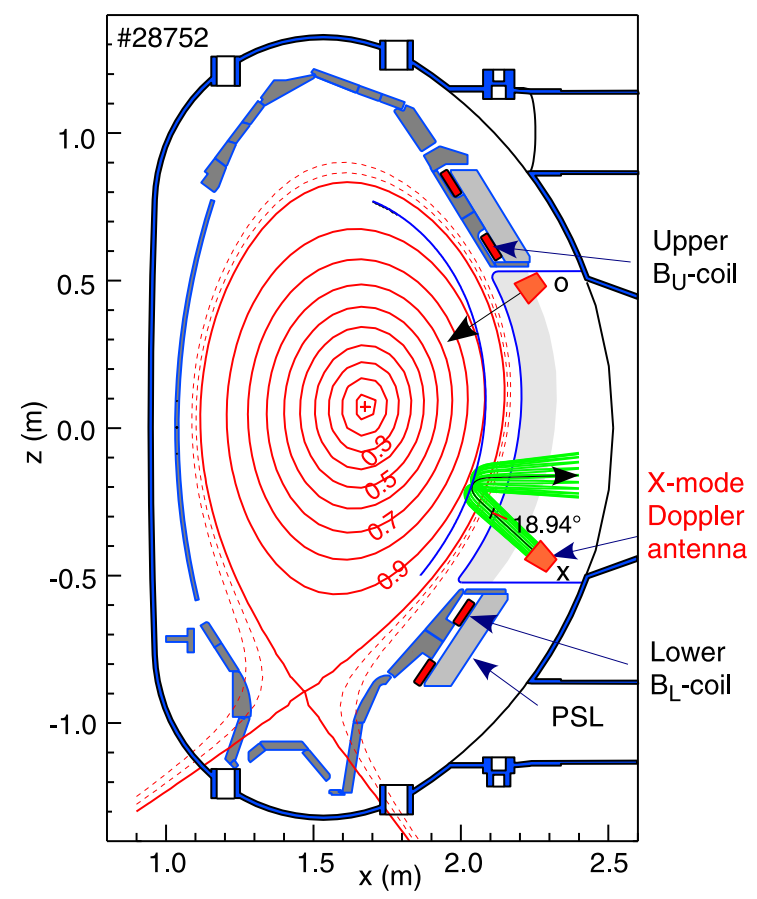

Figure 2. Poloidal cross section of the AUG vessel together with an axisymmetric flux surface plot. Marked are the locations of the upper and lower B-coils mounted on the passive stabilizing loops (PSL) together with the Doppler reflectometer antenna and a $64 \mathrm{GHz}$ probing beam trajectory for shot \#28752.

\section{Magnetic Perturbation (MP) coils}

AUG currently has installed sixteen in-vessel MP saddle coils in the form of two toroidal sets of eight coils (termed B-coils) above and below the tokamak low-field-side (LFS) mid-plane - spanning the full toroidal circumference, as shown in figure 1. A further eight "picture-frame" (A-coils) are also planned [19] for future installation. The coils are mounted on the upper and lower passive stabilizing loops (PSL) close to the plasma boundary, as shown in the poloidal cross-sectional view of the vessel in figure 2. Each coil consists of an encased, single 5-turn water cooled $\mathrm{Cu}$ conductor with separate coaxial feeders, which can be connected in (anti-)series to create MPs with toroidal mode numbers of $n=0,1,2$ and 4 . The MP poloidal mode number $m$ is defined by the coil's 
poloidal dimensions and their spacing etc. Generally, there is no single corresponding $m$ but a broad spectrum of modes and harmonics. The coils are powered by two independent supplies capable of generating $5 \mathrm{kAt}$, i.e. a maximum perturbation field $b_{r}$ of around $3 \mathrm{mT}$ at the plasma boundary in-front of an upper coil, which corresponds to a normalized perturbation field of $b_{r} / B \sim 10^{-3}$ for typical $-2.5 \mathrm{~T}$ operation.
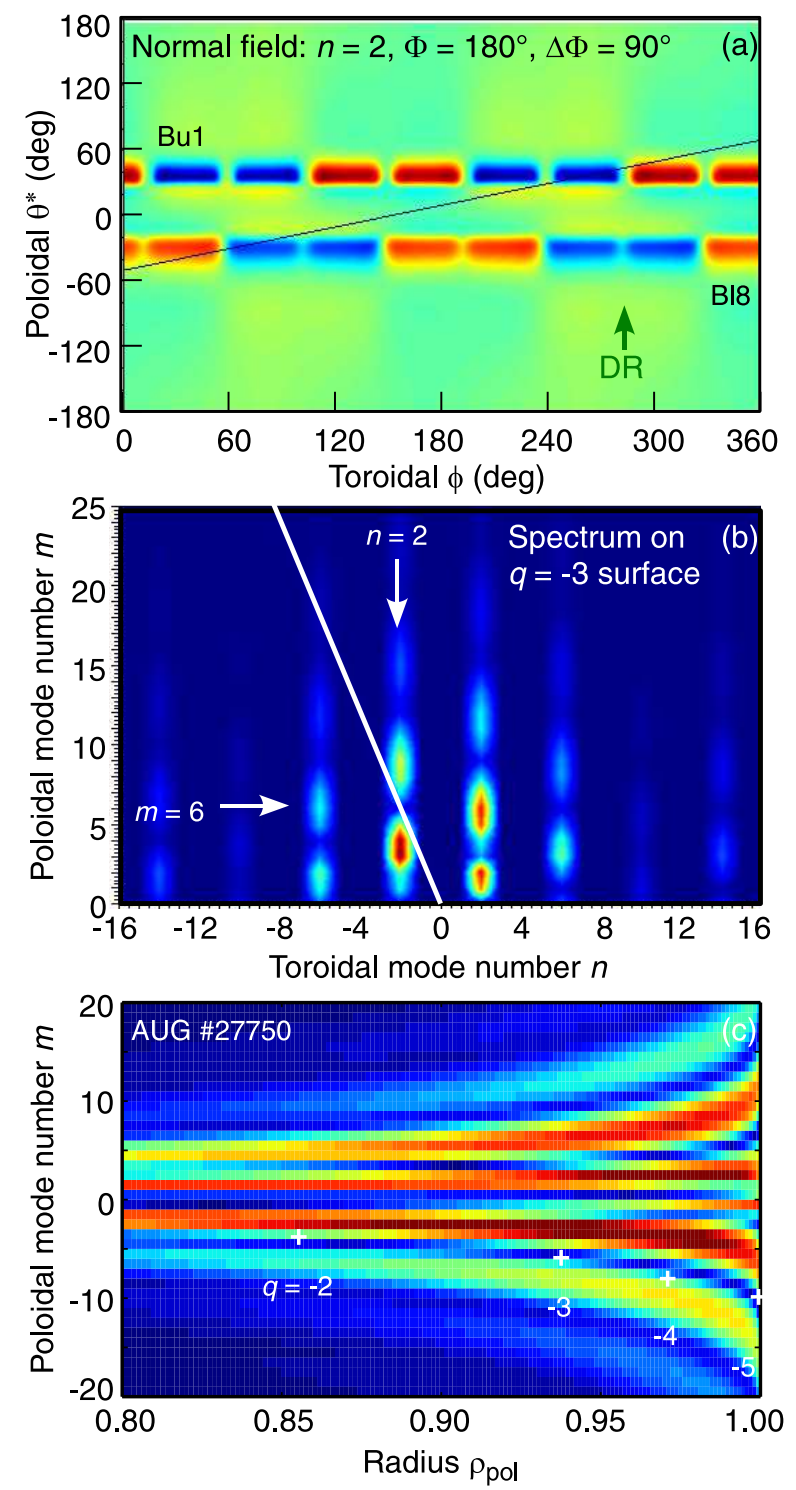

Figure 3. (a) MP vacuum field $b_{n}$ for non-resonant $n=2$ configuration on $q=-3$ surface (blue $=$ negative or inward pointing $b_{n}$, red $=$ positive) in straight field-line coordinates, (b) corresponding 2D Fourier $(m, n)$ spectrum, and (c) poloidal spectrum of $n=2$ component vs radius. White crosses mark rational $q$ surfaces.

For the static current results reported here, the toroidal phase $\Phi$ of the MP pattern relative to the tokamak can be varied in steps of $45^{\circ}$ for $n=1,90^{\circ}$ for $n=2$ and $180^{\circ}$ for $n=4$ by changing the current polarity of the individual coils via a switch-board panel. A half $\Phi$ step can also be achieved by powering only alternate coils. With programmed dynamic coil current waveforms it is also possible to simulate finer toroidal steps - but 
this was not used here. The relative phase $\Delta \Phi$ between the upper and lower coil set (i.e. coil parity) can also be varied to change the alignment of the MP field with respect to the tokamak field lines, and hence the degree of MP resonance with rational $q$ surfaces.

Figure 3(a) shows a contour plot of the vacuum, i.e. external, MP magnetic field $b_{n}$ normal to the flux surface (Biot-Savart equation; red is positive outward pointing $b_{n}$ ) [20] as a function of machine toroidal angle $\phi$ and poloidal straight field line angle $\theta^{*}$ calculated at the $q=-3$ surface for an $n=2 \mathrm{MP}$ configuration with $\Delta \Phi=90^{\circ}$ and $\Phi=180^{\circ}$ (here $\Phi=0$ is defined as a positive going $b_{n}$ with a null between coils $\mathrm{Bu} 8$ and $\mathrm{Bu} 1)$. The line indicates the field line inclination, which connects here opposite polarity coils in the upper and lower rows. The arrow indicates the Doppler reflectometer position. Below, in figure 3(b) is the corresponding 2D Fourier $(m, n)$ mode spectrum. The resonant spectral condition is marked by the inclined line (for the left-hand field line helicity $q<0$ ) which in this case does not intersect the main spectral peaks and thus indicates a non-resonant or non-field-aligned situation. Due to the low magnetic shear at the outboard mid-plane the MP will be simultaneously (non-)resonant for many edge rational surfaces [20], as shown by the poloidal mode $m$ spectra for the $n=2$ component as a function of poloidal flux radius $\rho_{\text {pol }}$ in figure $3(\mathrm{c})$. The crosses mark rational surface positions - which all lie in a trough (blue is low) in the spectral power. One side-effect of the plasma response to the MP field may be a modification of the resonance condition. However, in the absence of reliable modelling of the plasma screening for these discharges the resonance is discussed in terms of the vacuum component alone.

\section{Doppler reflectometer diagnostic}

An X-mode stepped frequency $(50-75 \mathrm{GHz})$ Doppler reflectometer [21] is used to measure the local radial electric field profile $E_{\mathrm{r}} \simeq-u_{\perp} B$ from the Doppler frequency shift $\omega_{\mathrm{D}}$ in the backscattered microwave beam (where $u_{\perp}=\omega_{\mathrm{D}} / k_{\perp}=v_{\mathrm{E} \times \mathrm{B}}+v_{\mathrm{ph}}$ and the usual condition of the turbulence phase velocity $\left.v_{\mathrm{ph}} \ll v_{\mathrm{E} \times \mathrm{B}} \& k_{\|} \ll k_{\perp}\right)$ [22]. In addition, the density fluctuation level $\left|\delta n_{\mathrm{e}}\left(k_{\perp}\right)\right|^{2}$ at the probed perpendicular wavenumber $k_{\perp}$ can be obtained from the integrated spectral peak $S\left(k_{\perp}\right)$. In the experiment the measurement location (beam turning point) and the probed $k_{\perp}=-2 N_{\perp} k_{\mathrm{o}}$ are obtained using a beam tracing code e.g. TORBEAM [23], with smooth density profiles fitted to experimental data and axisymmetric equilibria (i.e. without accounting for any $3 \mathrm{D}$ impact of the MP fields on the equilibrium). For typical L-mode conditions the diagnostic generally has a radial coverage from the far SOL to inside the edge gradient region, but at very low $n_{\mathrm{e}}$ the diagnostic can reach to around $\rho_{\text {pol }} \sim 0.6$ in radius.

The reflectometer bistatic antenna pair are located just above the lower B-coil row with an upward line-of-sight, as shown in the vessel cross-section in figure 2. Overlaid are flux surfaces and a $64 \mathrm{GHz} \mathrm{X}$-mode beam trace for a $n_{\mathrm{eo}}=2 \times 10^{19} \mathrm{~m}^{-3}, B_{\mathrm{t}}=-1.9 \mathrm{~T}$ shot, showing the Doppler reflectometer measures the LFS $E_{\mathrm{r}}$ poloidally somewhat below the magnetic axis. Toroidally the reflectometer probes between coils 6 and 7 (sector 13), as shown in the tokamak plan view of figure 4 . Thus, by rotating the MP coil phasing 


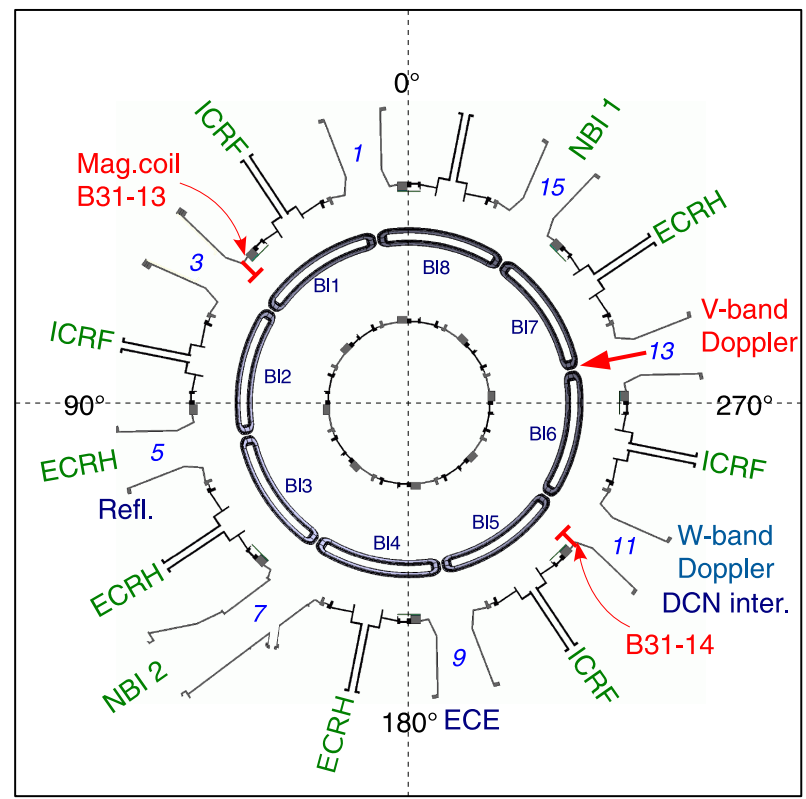

Figure 4. Schematic top view of the AUG vessel showing the toroidal locations of the B-coils with selected diagnostics and heating systems.

relative to the fixed diagnostic, the MP toroidal response may be investigated.

\section{Edge $E_{\mathrm{r}}$ response to $\mathrm{MP}$}

Figure 5 shows the response of various edge plasma profiles to the application of an $n=2$ odd-parity $\left(\Delta \Phi=180^{\circ}\right)$ edge "mostly resonant" MP for a typical $n_{\mathrm{eo}}=2 \times 10^{19} \mathrm{~m}^{-3}$, $B_{\mathrm{t}}=-2.5 \mathrm{~T}, I_{\mathrm{p}}=1.0 \mathrm{MA}, q_{95}=-4.2$, L-mode discharge. The schematic shows the MP configuration ( $b_{n}$ directions). With a (close to maximum) B-coil current of $I_{B}=0.9 \mathrm{kA}$ the MP impact is very pronounced in $E_{\mathrm{r}}$, figure $5(\mathrm{a})$ (red with and blue without MP) across both the edge gradient region (3) where the usual negative $E_{\mathrm{r}}$ well is inverted, and in the near SOL region (2) where there is a distinct reduction of the $E_{\mathrm{r}}$ peak just outside the separatrix. In the far SOL (1) there is little or no observable impact; and again inside of the edge gradient (4) where the $E_{\mathrm{r}}$ profiles re-converge. The $E_{\mathrm{r}}$ well reversal is consistent with TEXT results (HIBP) [7], Tore Supra (Scattering) [13], TEXTOR (reflectometer) [17] and (probes) [14] and MAST (probes) [12] - indicating that the Doppler reflectometer measurements are robust.

The usual explanation for the $E_{\mathrm{r}}$ well inversion is the ambipolar plasma response to the opening and/or mixing (stochastization) of the edge magnetic field lines resulting from the MP field penetration [7]. The creation of a stochastic field region in the edge is also indicated by Poincoré plots of the computed vacuum field [24], and by the field line connection length $L_{\mathrm{c}}$ (from inner to outer divertor), as shown in figure 5 (b). Without the MPs $L_{\mathrm{c}}$ is small in the SOL and infinity for the closed field line region (blue curve). With MPs the field lines inside the separatrix begin to break and $L_{\mathrm{c}}$ drops to around $1-2 \mathrm{~km}$ (red curves). The radial structure in $L_{\mathrm{c}}$ is on the mm scale - comparable to typical island 

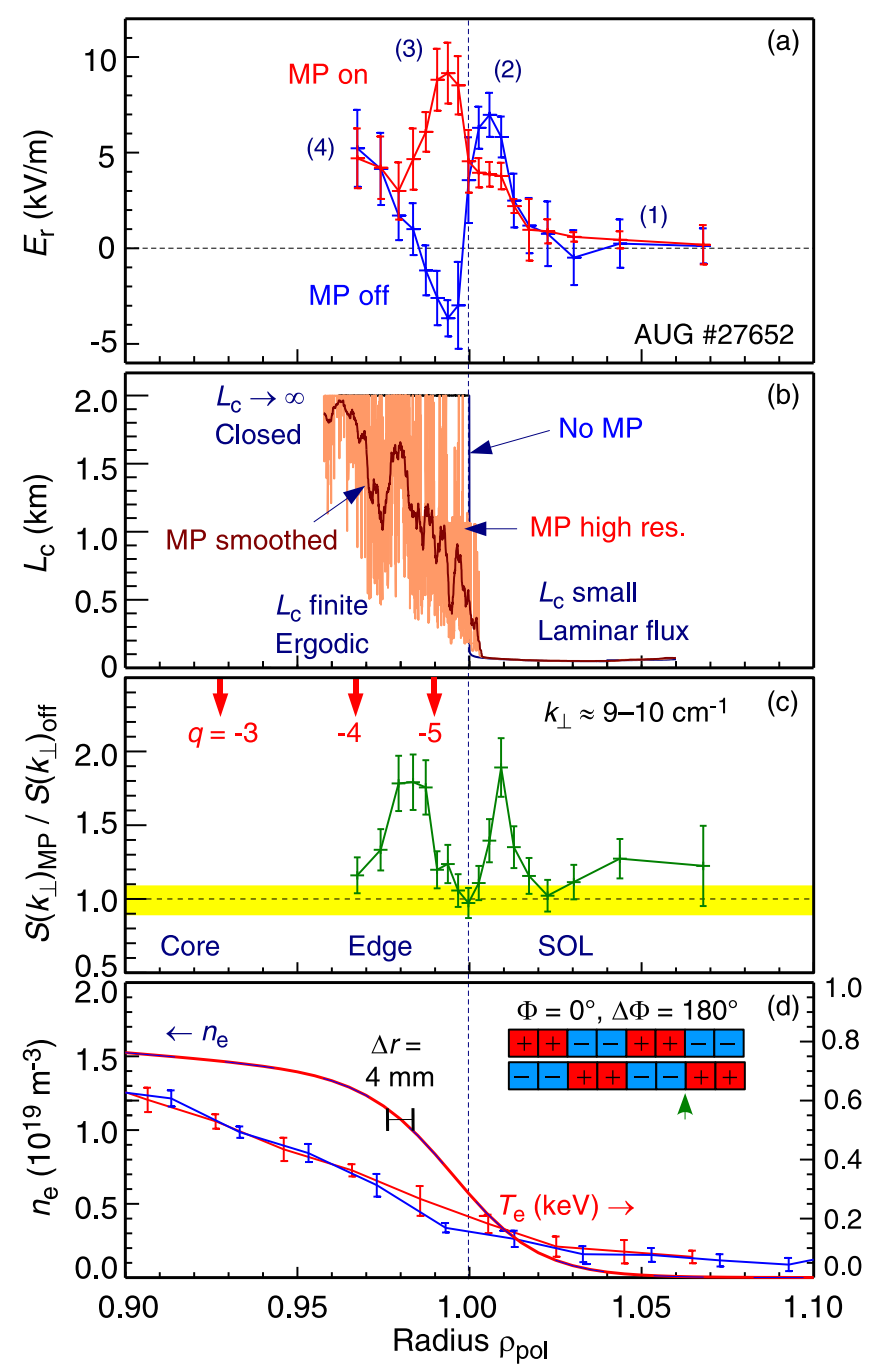

Figure 5. (a) L-mode $E_{\mathrm{r}}$, (b) vacuum connection length $L_{\mathrm{c}}$, (c) turbulence ratio, and (d) $n_{\mathrm{e}}$ and $T_{\mathrm{e}}$ radial profiles with (red) and without (blue) $n=2$, "resonant" $\left(I_{B}=0.9 \mathrm{kA}, \Phi=0^{\circ}, \Delta \Phi=180^{\circ}\right)$ odd-parity MPs $\left(-2.5 \mathrm{~T}, 1.0 \mathrm{MA}, q_{95}=-4.2\right.$, $\left.P_{\text {ech }}=0.7 \mathrm{MW}, T_{\text {eо }}=5 \mathrm{keV}, n_{\mathrm{eo}}=2 \times 10^{19} \mathrm{~m}^{-3}\right)$.

widths. Interpreting the width of the $E_{\mathrm{r}}$ inversion region (note the outer inversion radii is close to the computed last closed flux surface LCFS from the axisymmetric equilibrium) as an indication of the MP field penetration gives an estimated depth of $\sim 1.5 \mathrm{~cm}-$ which is somewhat narrower than the apparent vacuum field line mixing region from the $L_{\mathrm{c}}$ calculation, and is suggestive of non-negligible plasma screening effects.

Generally the L-mode edge density turbulence is spectrally broad-band - up to several hundred $\mathrm{kHz}$ and several $\mathrm{cm}^{-1}[25]$. Its response to the MP is shown in figure 5(c) in the form of a turbulence enhancement factor calculated from the integrated spectral Doppler intensity $S\left(k_{\perp}\right) \propto\left|\delta n_{\mathrm{e}}\right|^{2}$ (at the probed $k_{\perp} \sim 9 \mathrm{~cm}^{-1}$ - which is representative of the overall fluctuation behaviour) with MPs, normalized to the non-MP $S\left(k_{\perp}\right)$ (from the same discharge). In the two regions where the $E_{\mathrm{r}}$ profile is impacted the turbulence is also enhanced by a factor of 2 . In both the near SOL and in the edge gradient the 
peak turbulence enhancement aligns with regions where the negative $E_{\mathrm{r}}$ shear has either been reduced (at the SOL peak) or reversed (in the gradient region). Elsewhere, in the far SOL and around the separatrix, the turbulence level remains unaffected; which is generally consistent with far SOL probe measurements [16].

The corresponding kinetic profiles, electron temperature $T_{\mathrm{e}}$ from ECE and density $n_{\mathrm{e}}$ - in this case a modified hyperbolic tangent fitted to Lithium beam, Thomson Scattering, and FMCW reflectometry data (which describes well the edge $n_{\mathrm{e}}$ profile shape) are shown in figure $5(\mathrm{~d})$. There is little change in the kinetic profiles with the MPs (within measurement errors) and no evidence of any significant edge density pumpout associated with the MPs at these low densities in L-mode. However, the far SOL does show a slight $n_{e}$ enhancement together with divertor strike-point splitting [15]. $T_{\mathrm{i}}$ measurements are unfortunately not available in these experiments.

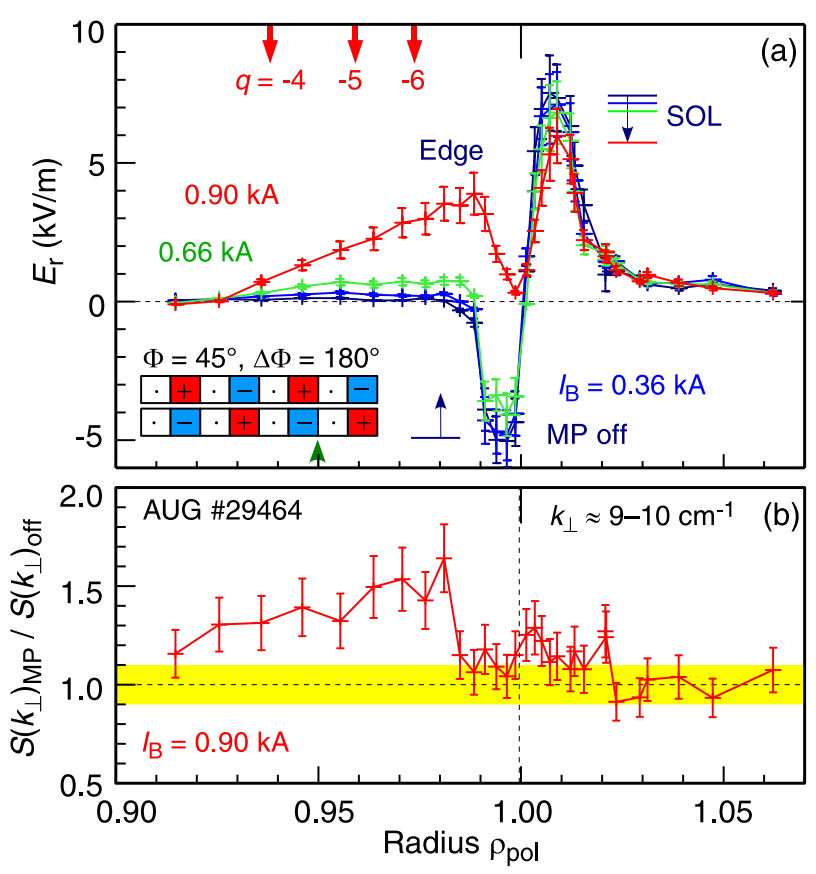

Figure 6. (a) Edge $E_{\mathrm{r}}$ profiles at fixed L-mode plasma parameters $(-2.5 \mathrm{~T}, 0.8 \mathrm{MA}$, $\left.q_{95}=-5.2, P_{\text {ech }}=0.55 \mathrm{MW}, T_{\mathrm{eo}}=4.5 \mathrm{keV}, n_{\mathrm{eo}} \approx 1.5 \times 10^{19} \mathrm{~m}^{-3}\right)$ with $n=2$, "resonant" $\left(\Phi=45^{\circ}, \Delta \Phi=180^{\circ}\right)$, odd-parity, MP $I_{B}$ current steps; (b) turbulence enhancement factor for $I_{B}=0.9 \mathrm{kA}$ MP step.

\section{MP thresholds}

It has long been understood that the plasma attempts to resist the penetration of the externally applied MP field via the formation of shielding currents [26]. However, the plasma screening is difficult to calculate and is a topic of much theoretical study $[27,28,29,30,31,32,33]$. For the low density, low-rotation, L-mode conditions of interest here calculations of the plasma screening are currently not available. But, it is known that screening leads to a threshold or critical field for the MP penetration. This has been investigated for different collisionality regimes, but generally scales linear with 
$n_{\mathrm{e}}$ and inverse with $B_{\mathrm{t}}$ [34]. A critical field of $\left(b_{n} / B\right)_{\text {crit }} \sim \mathcal{O}\left(10^{-4}\right)$ is typically expected for usual tokamak conditions. Experimentally, clear threshold effects have been observed in MAST [12], but are less clear in other machines [7, 11, 14, 35]. Density and plasma rotation dependences have also been reported [13, 27].

Figure 6 shows such a critical threshold in the $E_{\mathrm{r}}$ for an $n=2$ edge "resonant" MP. The MP coil current is stepped from 0 to $0.9 \mathrm{kA}$ at constant density and electron temperature [shown in figure $11(\mathrm{e})]$ and energy confinement. Both the near SOL $E_{\mathrm{r}}$ peak and the edge gradient $E_{\mathrm{r}}$ well begin to respond slowly to the increasing $I_{B}$, but it is only at the highest current (red curve) that the $E_{\mathrm{r}}$ well rises sharply and the SOL peak drops (outside of error bars). The turbulence is again enhanced in regions of reduced negative $E_{\mathrm{r}}$ shear - particularly inside of the $E_{\mathrm{r}}$ well where the shear becomes positive.
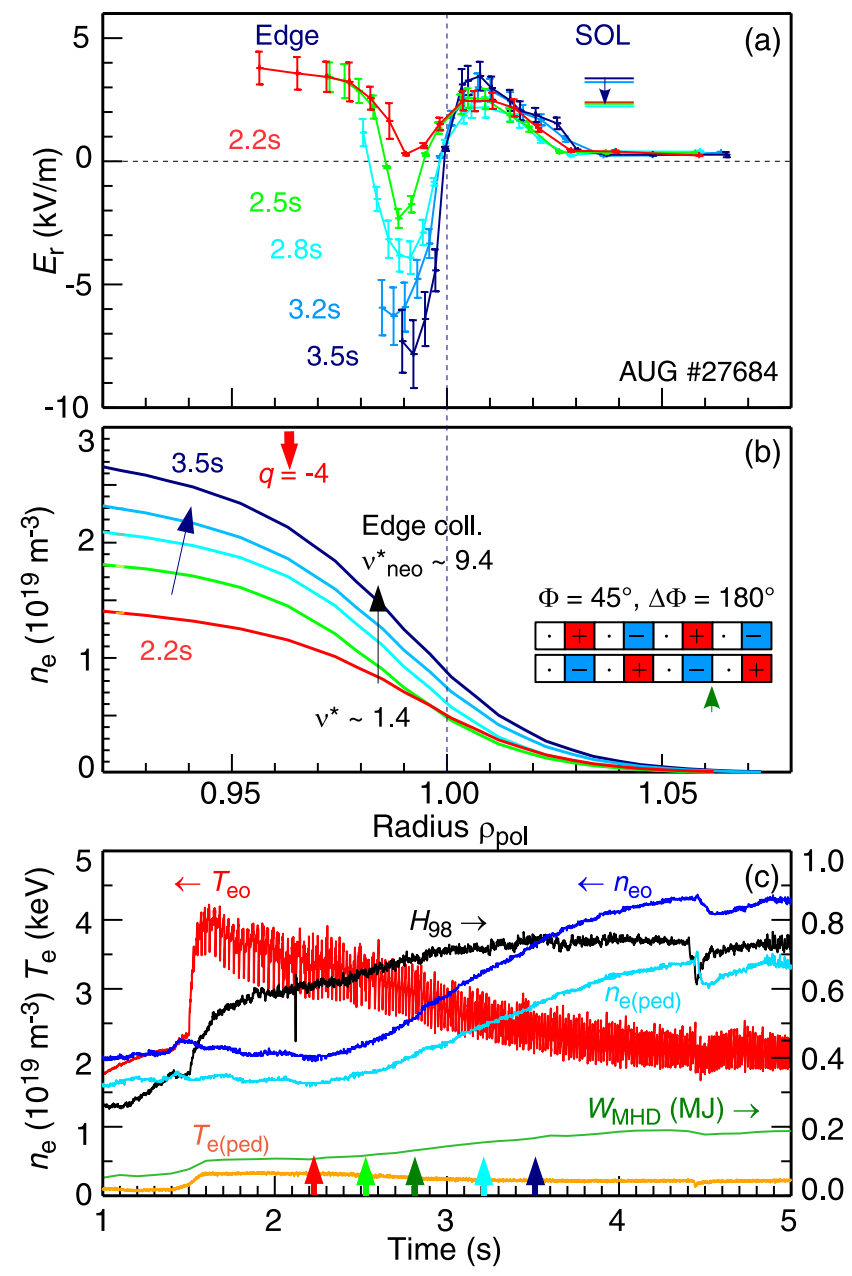

Figure 7. (a) Edge $E_{\mathrm{r}}$, (b) $n_{\mathrm{e}}$ profiles and (c) time traces of $n_{\mathrm{e}}, T_{\mathrm{e}}$ etc. during an L-mode density ramp with $n=2$ "resonant" $\left(I_{B}=0.9 \mathrm{kA}, \Phi=45^{\circ}, \Delta \Phi=180^{\circ}\right)$ odd-parity MPs. $\left(-2.5 \mathrm{~T}, 1.0 \mathrm{MA}, q_{95}=-4.3, P_{\text {ech }}=0.5 \mathrm{MW}\right.$.)

The expected density dependence of the MP threshold is displayed in the edge $E_{\mathrm{r}}$ profile evolution in figure 7 (a) during an L-mode density ramp of $n_{\mathrm{eo}}=2 \rightarrow 5 \times 10^{19} \mathrm{~m}^{-3}$ with a fixed $I_{B}=0.9 \mathrm{kA}$ and an $n=2$ edge "resonant" MP configuration. As 
$n_{\mathrm{e}}$ rises, $T_{\mathrm{e}}$ falls, figure 7 (b) \& (c), (with a corresponding increase in collisionality $\left.\nu_{\text {neo }}^{*}=q_{95} R_{o} \epsilon^{-3 / 2} \lambda_{\mathrm{e}}^{-1}\right)$ the MP impact disappears, both in the near SOL where the $E_{\mathrm{r}}$ peak flips between reduced and non-reduced states when $n_{\mathrm{e}}$ exceeds a certain value (compare curves for 2.8 and $3.5 \mathrm{~s}$ ); and in the $E_{\mathrm{r}}$ well, which conversely gradually reverts to the no-MP depth with increasing $n_{\mathrm{e}}$. Note that without MPs the variation in $E_{\mathrm{r}}$ with density is less than $1 \mathrm{kVm}^{-1}$. The different density responses for the SOL (threshold) and $E_{\mathrm{r}}$ well regions (gradual transition) suggests distinct MP mechanisms for the laminar SOL and stochastic edge regions. The absence of an MP impact at high density on $T_{\mathrm{i}}$ and the $E_{\mathrm{r}}$ well has also been observed using CXRS [18].

\section{MP resonance}

The degree of MP resonance is defined as the alignment of the perturbation field with the equilibrium rational surface field lines. This can be varied via the plasma safety factor $q$ profile (i.e. via $B_{\mathrm{t}}$ or $I_{p}$ ), or by changing the toroidal and poloidal spectra of the MP field. For a selected $n$ configuration this can be achieved by shifting the relative $\Delta \Phi$ phase between the upper and lower B-coil sets [19].

Figure 8 compares the edge $E_{\mathrm{r}}$ (top row, blue without and red with MP) and turbulence behaviour (middle row) from very similar $n_{\mathrm{eo}} \approx 1.3 \times 10^{19} \mathrm{~m}^{-3}$, L-mode discharges for three possible differential phases: (a) $\Delta \Phi=0^{\circ}$ (even parity), (b) $90^{\circ}$ (mid parity) and (c) $180^{\circ}$ (odd parity) for an $n=2 \mathrm{MP}$ with $\Phi=0^{\circ}$. The bottom row of figure 8 shows the corresponding MP poloidal vacuum spectra for each configuration and shows how changing the differential phase changes the MP spectral components $(n=2, m=8 \& 10)$ from moderate, to weak, to strong at the $q=-4\left(\rho_{\mathrm{pol}} \approx 0.98\right)$ and $q=-5\left(\rho_{\mathrm{pol}} \approx 0.99\right)$ surfaces respectively. All three parities display some degree of $E_{\mathrm{r}}$ impact, the weakest being for the "non-resonant" case and the strongest for the "mostly resonant" case. The turbulence enhancement only being significantly enhanced in the most resonant configuration around the $E_{\mathrm{r}}$ reversal, again associated with the region of negative $E_{\mathrm{r}}$ shear loss.

Figure 9 shows the effect of changing the $q$ profile. The edge $E_{\mathrm{r}}$ profiles are for a pair of matched $-2.5 \mathrm{~T}$ L-mode discharges at $I_{\mathrm{p}}=1.0$ (red/solid) and $0.6 \mathrm{MA}$ (blue/dashed) with $\left.n_{\mathrm{eo}} \approx 1.5 \times 10^{19} \mathrm{~m}^{-3}\right)$ and $n=2$ edge "resonant" $\left(I_{B}=1.0 \mathrm{kA}\right.$, $\Phi=45^{\circ}, \Delta \Phi=180^{\circ}$ ) MP. For the $1.0 \mathrm{MA}$ case the $E_{\mathrm{r}}$ well is strongly impacted, but is unaffected for the 0.6 MA case. Below in figure 9(b) are the corresponding $q$ profiles from two equilibrium reconstruction codes; FPP and CLISTE. For $I_{\mathrm{p}}=1.0 \mathrm{MA}$ the $q=-4$ and -5 rational surfaces are close to the edge and accessible to the MP field. The MP vacuum poloidal spectrum in figure 9 (c) confirms that the $(n=2, m=10)$ spectral component is significant at the $q=-5$ surface. Lowering the $I_{\mathrm{p}}$, raises the edge $q$ profile, such that the low order rational surfaces are now much deeper and the edge is filled with high order surfaces. The $(m=10 @ q=-5)$ component is much reduced (hardly resonant) and the higher $(m=14 @ q=-7$ and $m=16 @ q=-8)$ components are negligible. 

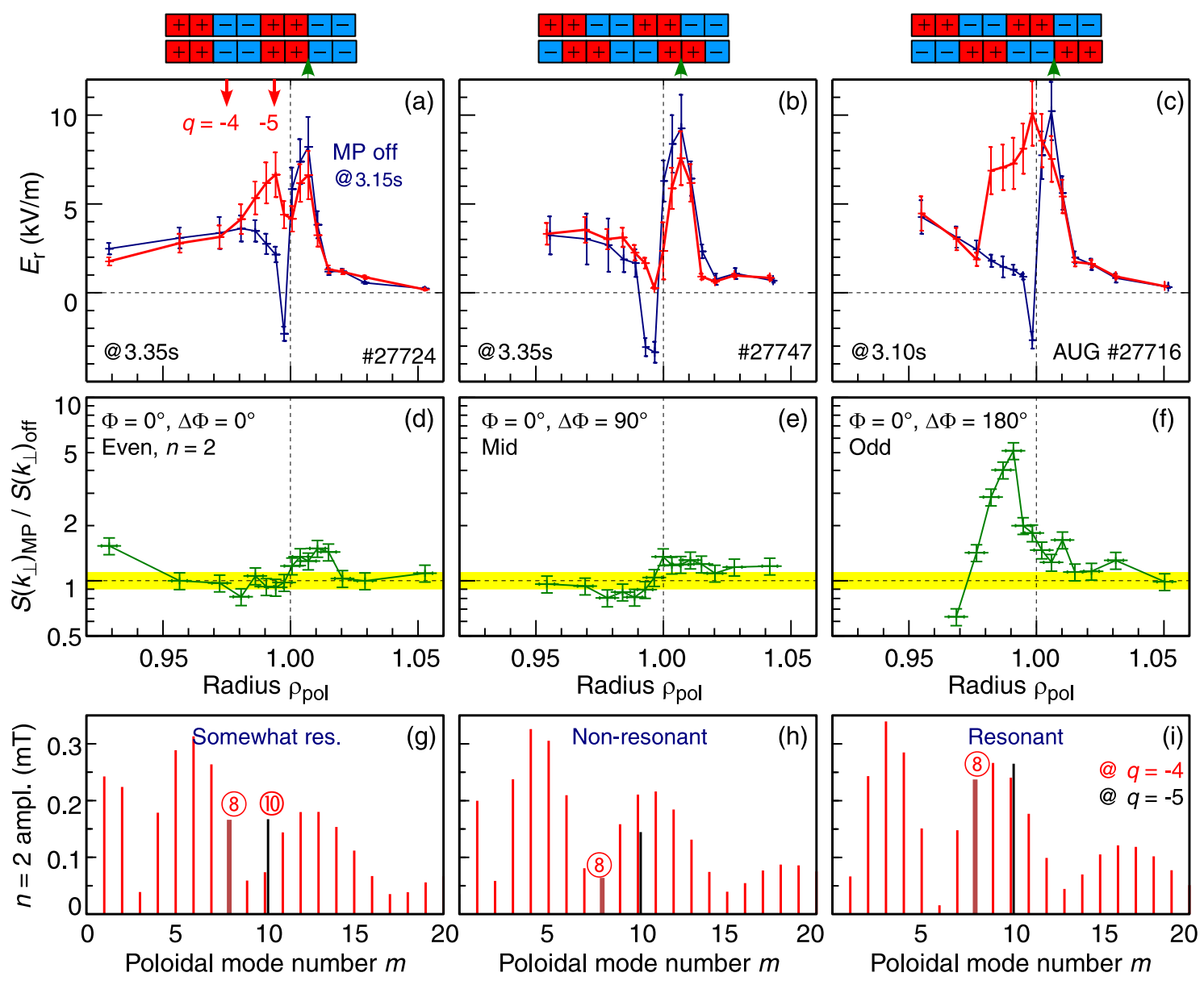

Figure 8. Top: $E_{\mathrm{r}}$ profiles, middle: turbulence ratio, bottom: MP m-spectra from matched L-mode shots $\left(-2.3 \mathrm{~T}, 1.0 \mathrm{MA}, q_{95} \approx-4, P_{\text {ech }}=0.6 \mathrm{MW}, T_{\mathrm{eo}}=4.5 \mathrm{keV}\right.$, $\left.n_{\mathrm{eo}} \approx 1.3 \times 10^{19} \mathrm{~m}^{-3}\right)$ with $n=2\left(I_{B}=0.9 \mathrm{kA}, \Phi=0^{\circ}\right)$ MPs for (a) $\Delta \Phi=0^{\circ}$ (even), (b) $\Delta \Phi=90^{\circ}$ (mid) and (c) $\Delta \Phi=180^{\circ}$ (odd).

\section{MP toroidal phase}

The MP field is helical in form with both toroidal and poloidal structure. This structure can be sampled by shifting the MP coil phase, i.e. a rigid toroidal rotation of the MP field, relative to the fixed diagnostic measurement point. The poloidal structure, however, is less easy to access with only two poloidal coil rows and one measurement location.

The toroidal variation is illustrated in figure 10 with a fixed $n=2, \Delta \Phi=90^{\circ}$ edge "non-resonant" configuration for four $\Phi=0,90,180,270^{\circ}$ static MP phases during a single discharge with constant edge $n_{\mathrm{e}}$ and $T_{\mathrm{e}}$ etc. Both the near SOL $E_{\mathrm{r}}$ and the edge $E_{\mathrm{r}}$ well clearly display individual reactions to the MP orientation - which is particularly evident in the $\Phi=180^{\circ}$ and $270^{\circ}$ orientations. This suggests separate, but not necessarily unrelated, toroidal 3D structures for the SOL (laminar) and edge (stochastic) regions.

As in previous examples the MP affected $E_{\mathrm{r}}$ regions are also accompanied by changes in the density turbulence level, figure 10 (bottom row). However, in this "non- 

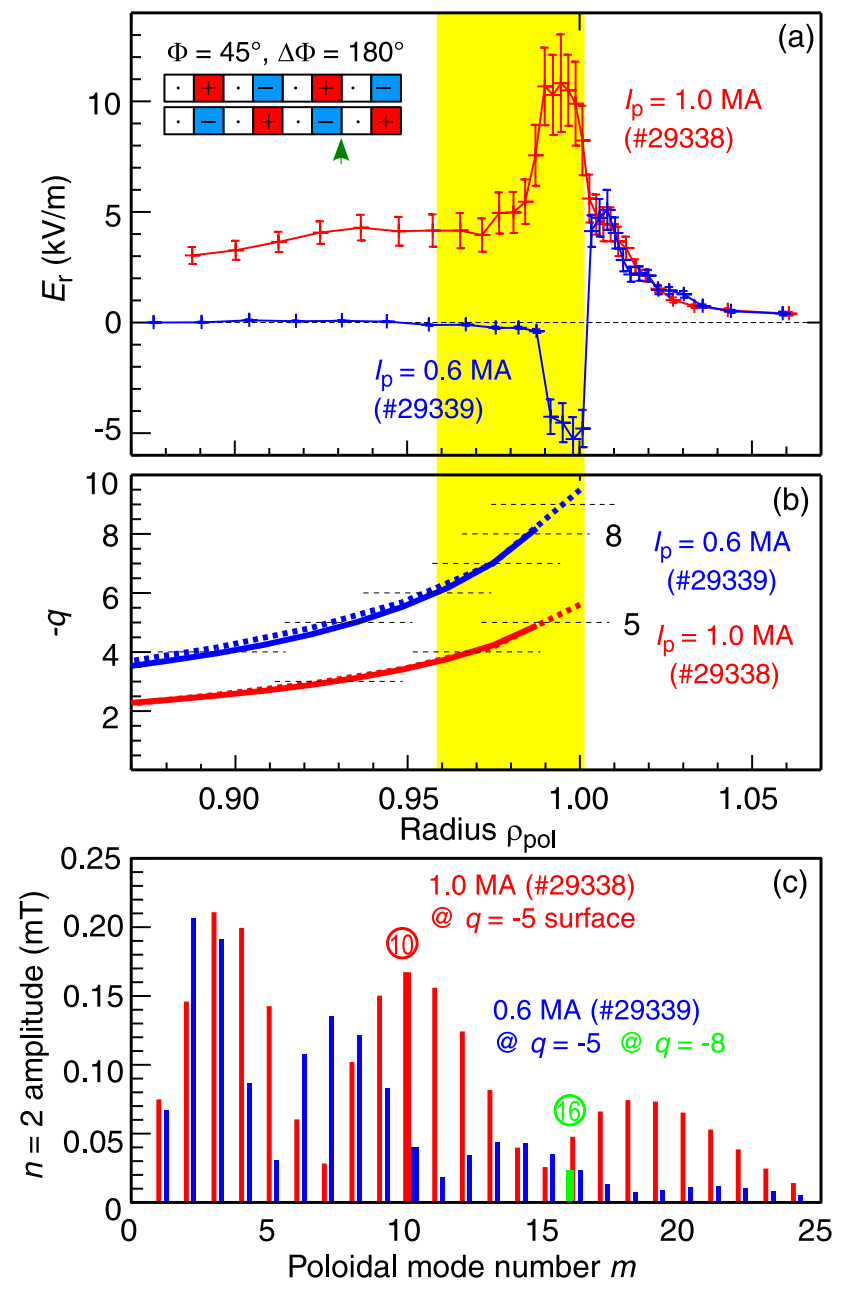

Figure 9. (a) $E_{\mathrm{r}}$, (b) $q$-profiles, and (c) poloidal mode spectra for matched L-mode (red) $I_{\mathrm{p}}=1.0 \mathrm{MA}$ and (blue) $0.6 \mathrm{MA}$ shots $\left(-2.5 \mathrm{~T}, P_{\text {ech }}=0.6 \mathrm{MW}, T_{\mathrm{eo}} \approx 5 \mathrm{keV}\right.$, $\left.n_{\mathrm{eo}} \approx 1.5 \times 10^{19} \mathrm{~m}^{-3}\right)$ with odd-parity $n=2, I_{B}=1.0 \mathrm{kA}, \Phi=45^{\circ}, \Delta \Phi=180^{\circ} \mathrm{MPs}$.

resonant" MP configuration only the near SOL shows significant (factor 2) enhancements in the turbulence level when the SOL $E_{\mathrm{r}}$ peak is suppressed. In the edge gradient region the turbulence level is actually decreased. Contrasting this behaviour with figures $5(\mathrm{c})$, 6(b) and 8(f): all resonant, with figure 8(e): non-resonant, suggests that the edge region $\delta n_{\mathrm{e}}$ may be increased (by a factor of 10 for strongly resonant MPs) or decreased depending on the MP configuration.

In principle, rotating the MP phase $\Phi$ should not affect the MP spectra or degree of resonance. However, observations with $n=1$ MPs [36, 37] as well as recent measurements with $n=2$ indicate that certain $\Phi$ can either promote or hinder mode locking, implying toroidal asymmetries or subtle interactions between the MP and intrinsic static fields, such as the presence of an $n=2$ error field. Note that lockmode cases are explicitly excluded in this paper.

3D vacuum field-line tracing including MPs predict a toroidally sinusoidal radial displacement of the low-field-side separatrix position [38]. The displacement amplitude 

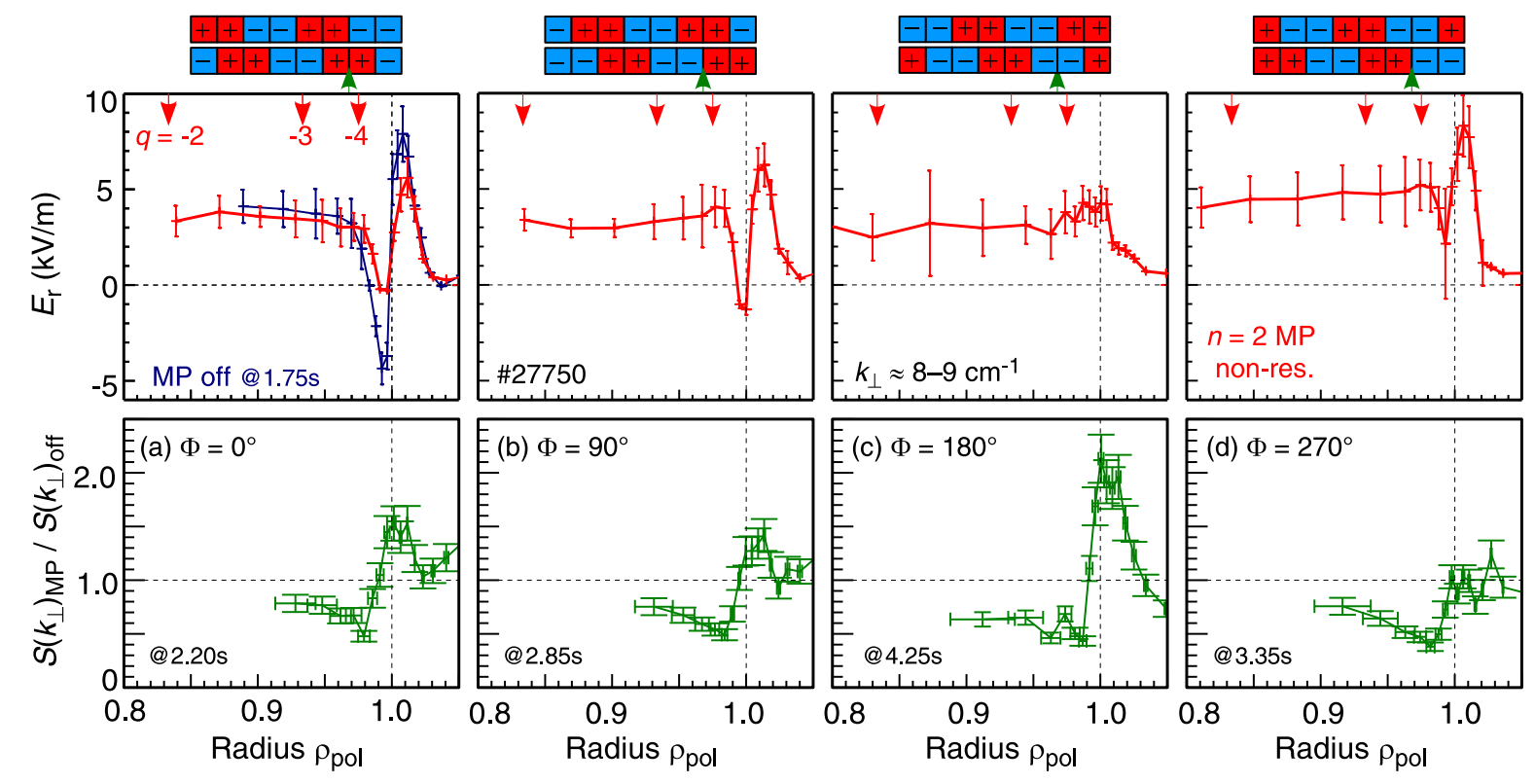

Figure 10. (Top row) $E_{\mathrm{r}}$ profiles and (bottom row) turbulence enhancement factor with (red) \& without (blue) $n=2$, "non-resonant" ( $\left.I_{B}=0.9 \mathrm{kA}, \Delta \Phi=90^{\circ}\right)$ MPs for $\Phi=0^{\circ}, 90^{\circ}, 180^{\circ}$ and $270^{\circ}$ toroidal phases. $\left(-2.5 \mathrm{~T}, 1.0 \mathrm{MA}, q_{95} \approx-4\right.$, $P_{\text {ech }}=0.65 \mathrm{MW}$, with constant $T_{\mathrm{eo}}=4.5 \mathrm{keV}$ and $\left.n_{\mathrm{eo}} \approx 1.5 \times 10^{19} \mathrm{~m}^{-3}\right)$.

depends strongly on the MP configuration but, can be several mm in worst cases - leading to profile mapping issues $[39,40]$. Compression of the flux surfaces is not critical for the Doppler reflectometer measurement position since it follows the correspondingly displaced density profile, which is seen to remain roughly constant in the flux surface coordinate $\rho_{\text {pol }}$ (ignoring small changes in $B(R)$ for X-mode probing). A $\Delta r \sim 4 \mathrm{~mm}$ bar (maximum separatrix displacement at the diagnostic location) is shown for comparison in figure $5(\mathrm{~d})$ - which is comparable in magnitude to the Doppler beam scattering width, i.e. measurement error. This allows, with care, the use of the unperturbed (nonMP) density profile and an axisymmetric equilibria in the beam tracing procedure for the Doppler reflectometer analysis. Plasma screening may of course introduce localized non-uniformities in the edge equilibrium, which are not accounted for here. Flux surface distortion will, however, affect the potential gradient, e.g. $E_{\mathrm{r}}$, to a small degree.

\section{Edge flow perturbation}

Geodesic Acoustic Modes (GAMs) are universally observed in the edge confinement region of AUG low collisionality L-mode discharges, cf. [41]. Figure 11(a \& b) shows the effect of applying an edge "resonant" $n=2 \mathrm{MP}$ on the $E_{\mathrm{r}} \times B$ flow and $n_{\mathrm{e}}$ fluctuation spectra at the GAM maximal location $\left(\rho_{\text {pol }} \approx 0.99\right)$ for the discharge shown in figure 6 . For the last $I_{B}$ step with the impacted $E_{\mathrm{r}}$ well, the coherent flow spectral peak is upshifted in frequency from $14.6 \mathrm{kHz}$ to $15.9 \mathrm{kHz}$ and weakened in amplitude. Overall, $\delta E_{\mathrm{r}}$ falls while the $\delta n_{\mathrm{e}}$ spectra is broadly enhanced. The non-MP GAM peak generally 
extends from the separatrix across the negative $E_{\mathrm{r}}$ shear region, increasing in frequency with temperature in a staircase fashion. Each frequency stair corresponding to a GAM zonal layer [41]. The GAM peaks in magnitude close to the stair inner edge. With MPs the mode extends somewhat deeper, but peaks further out, closer to the $E_{\mathrm{r}}$ minimum.
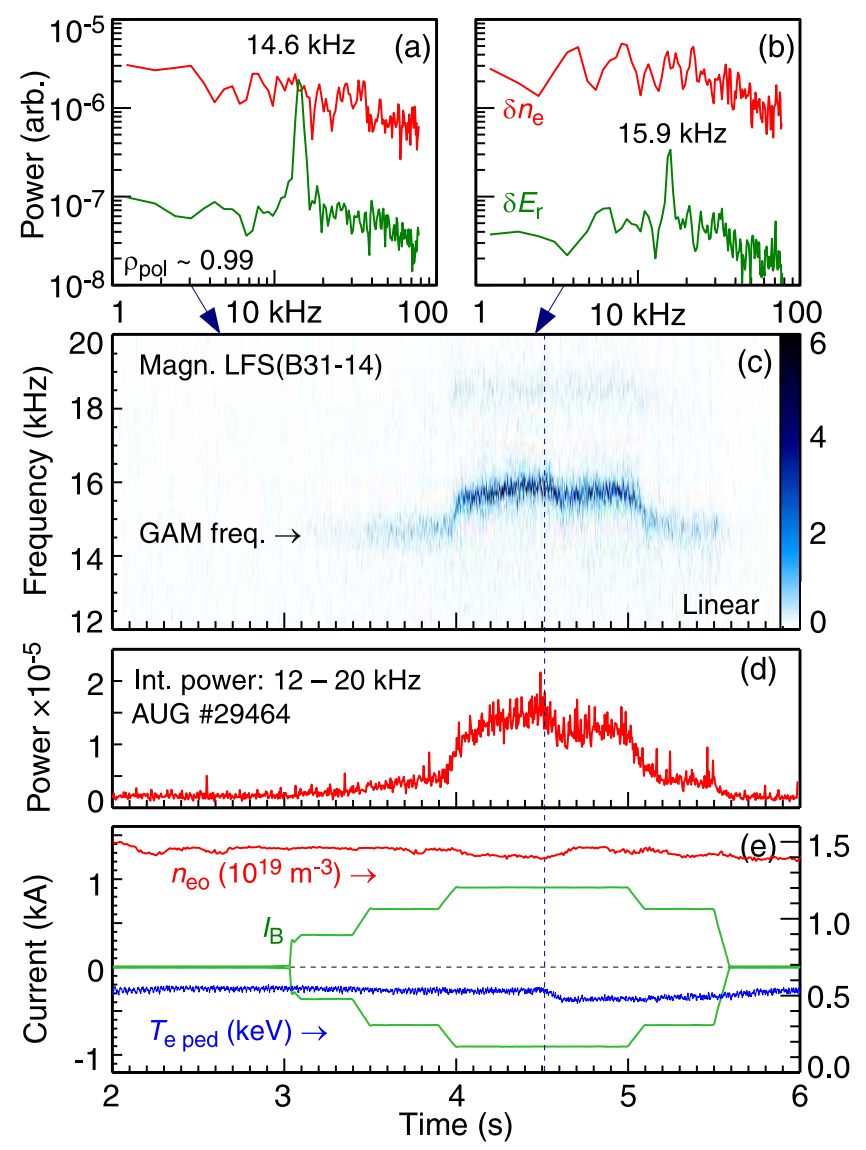

Figure 11. $E_{\mathrm{r}}$ and $n_{\mathrm{e}}$ spectra at $\rho_{\mathrm{pol}} \approx 0.99$ without (a) and with (b) MP, (c) LFS Mirnov (B31-14) spectrogram with linear power scale, (d) $12-20 \mathrm{kHz}$ integrated spectral power, and (e) core $n_{\mathrm{eo}}$, edge $T_{\text {edge }}$ and MP current $I_{B}$ time traces for L-mode discharge \#29464. Parameters as per figure 6.

Accompanying the up-shifted flow perturbation is a corresponding magnetic perturbation, as illustrated in figure $11(\mathrm{c} \& \mathrm{~d})$ with the spectrogram of a LFS midplane Mirnov coil $\delta b_{\mathrm{r}}$ signal and the $12-20 \mathrm{kHz}$ band-limited spectral power. A weak magnetic signature appears in the penultimate $I_{B}$ step at the GAM frequency and then smoothly transitions in frequency and amplitude with the full MP $I_{B}$ step. Note there is no corresponding rise in the edge $T_{\mathrm{e}}$ to account for a classic GAM frequency jump. $T_{\mathrm{i}}$ was not measured here, but would need to jump by $>30 \%$ to account for the frequency step. GAMs in AUG generally display no $\delta b_{\mathrm{r}}$ signature, but often a $\delta b_{\text {pol }}$ component [42] with a not too dissimilar to the predicted [43] and measured $m= \pm 2$ structure in TCV $[44]$.

There is extensive theory on the penetration of resonant MP components leading to island formation at resonant rational surfaces and seeding neoclassical tearing modes 
(NTMs), as well as inducing toroidal torques etc. cf. [34, 32]. In the case of MHD modes reacting to MPs in AUG they are seen to slow down, and eventually lock [45]. However, in the $n=2$ cases discussed here the mode spins-up in frequency with the application of sufficient $I_{B}$. Although there is a minor burp in frequency (vertical line) in figure $11(\mathrm{c})$ coinciding with a $T_{\mathrm{e}}$ dip is hard to distinguish cause or effect. In addition, signatures remain even when clear mode-locking has taken place. On MAST, similar frequency up-shifting of flow perturbations were observed using probes [46]. As there was no magnetic signature (evidence against tearing modes) they were identified as GAMs with the frequency shift attributed to an increase in the edge plasma Mach number.

The AUG data suggests that the GAM is structurally modified by the MP. The $\delta b_{\mathrm{r}}$ signatures have an $(n=2, m>7)$ structure, but the toroidal behaviour is complex with a strong dependence on the MP configuration and phase $\Phi$. This suggests the intrinsic $(n=0)$ GAM $b$-field interacts with the applied MP $b_{n}$ field creating a non-axisymmetric $(n \neq 0)$ GAM and the observed $\delta b_{\mathrm{r}}$ signature. The GAM is weakened and radially eroded by the progressive field-line stochatization of the edge (not inconsistent with TEXTOR long-range correlation results [47]). However, further measurements and modeling are required to establish the extent of stochasticity needed to destroy the GAM.

\section{Summary \& Discussion}

The high resolution AUG Doppler reflectometer results presented here both support previous observations as well as reveal new features. Specifically the reduction and reversal of the $E_{\mathrm{r}}$ well, and the dependence on $n_{\mathrm{e}}$ and $b_{n}$ thresholds linked to the MP penetration are identified. Different threshold dependencies for the SOL and confinement regions are also noted. Such differences may be attributed to the laminar vs stochastic field line nature of the two regions.

$n=2 \mathrm{MP}$ penetration into the confinement region appears to be effective only at relatively low plasma density, and in general extends only a few $\mathrm{cm}$ beyond the separatrix. However, at very low density and/or for $n=1 \mathrm{MPs}$ the penetration can be significant and the promotion of mode locking for certain MP phases is pronounced. Between mode locking and total field exclusion is a density range where much detailed information on the MP field structure and profile impact can be obtained. In this operational range, despite the evident impact on the edge $E_{\mathrm{r}}$ and turbulence, the MPs only mildly affect the kinetic profiles and energy confinement.

For the confinement region, the MP spectral content and degree of resonance with the edge rational surfaces plays a significant role. A high degree of MP resonance has a stronger impact with larger and wider $E_{\mathrm{r}}$ well reversals. The radial limit of the MP field penetration is particularly well illustrated when raising the $q$ profile and filling the edge with high order rational surfaces. To properly quantify the MP penetration, and the extent of the plasma response in modifying the resonance condition, requires modelling of the AUG geometry and L-mode conditions. This is now beginning. Likewise, the effect of plasma rotation on screening is also a topic of further investigation. 
The 3D structure of the MP field has also been probed by rotating the MP coil phase toroidally. Significant variations, as well as discrepancies, are revealed in the toroidal pattern in both the near SOL and the confinement regions - which again highlights the different nature of the two regions. The spatial sampling of the toroidal structure was limited here (four $\Phi$ for $n=2$ ), however, finer toroidal resolution measurements using dynamic coil currents have recently been achieved (to be presented shortly).

The turbulence behaviour is more complicated. $\delta n_{\mathrm{e}}$ is generally enhanced in the near SOL for all MP configurations, but can be enhanced or reduced across the edge depending on the degree of MP resonance and/or configuration. Nevertheless, there is a clear linkage between the $\delta n_{\mathrm{e}}$ behaviour and the negative $E_{\mathrm{r}}$ shear. Coincident with the $E_{\mathrm{r}}$ impact, the edge flow (GAM) properties are also modified. A coherent flow perturbation continues to exist across the edge region with a frequency which chirps up from the preceding GAM. The flow perturbation amplitude decreases, but is accompanied by an enhanced magnetic signature, consistent with an non-axisymmetric GAM. Finally, although only results for $n=2 \mathrm{MPs}$ were shown, similar overall behaviour is also observed for $n=1$ and $n=4 \mathrm{MPs}$, which will be presented in future work.

\section{Acknowledgments}

This work has been carried out within the framework of the EUROfusion Consortium and has received funding from the European Unions Horizon 2020 research and innovation programme under grant agreement number 633053. The views and opinions expressed herein do not necessarily reflect those of the European Commission. This work has also be performed in the framework of the Helmholtz Virtual Institute on plasma dynamical processes and turbulence using advanced microwave diagnostics.

\section{References}

[1] Evans T.E. et al. 2005 Nucl. Fusion 45225004

[2] Kirk A. et al. 2010 Nucl. Fusion 50034008

[3] Canik J.M. et al. 2010 Phys. Rev. Lett. 104045001

[4] Suttrop W. et al. 2011 Phys. Rev. Lett. 106225004

[5] Jeon Y.M. et al. 2012 Phys. Rev. Lett. 109035004

[6] Wootton A.J. 1990 J. Nucl. Mat. 176-177 77

[7] Yan X.Z. et al. 1991 Phys. Fluids B 33448

[8] Shoji T. et al. 1992 J. Nucl. Mat. 196-198 296

[9] McKee G.R. et al. 2013 Nucl. Fusion 53113011

[10] Xu Y. et al. 2006 Phys. Rev. Lett. 97165003

[11] Unterberg B. et al. 2007 J. Nucl. Mat. 363-365 698

[12] Tamain P. et al. 2010 Plasma Phys. Control. Fusion 52075017

[13] Payan J. et al. 1995 Nucl. Fusion 351357

[14] Xu Y. et al. 2007 Nucl. Fusion 471696

[15] Müller H.W. et al. 2013 J. Nucl. Mat. 438564

[16] Müller H.W. et al. 2014 Contrib. Plasma Phys. 54261

[17] Krämer-Flecken A. et al. 2006 Nucl. Fusion 46 S730 
[18] Viezzer E. et al. 2013 Nucl. Fusion 53053005

[19] Suttrop W. et al. 2009 Fusion Eng. Des. 84290

[20] Suttrop W. et al. 2012 Proc. 39th EPS Conf. (Stockholm) ECA 36F P2.092

[21] Conway G.D. et al. 2004 Plasma Phys. Control. Fusion 46951

[22] Conway G.D. et al. 2010 Plasma Fusion Res. 5 S2005

[23] Poli E. et al. 2001 Comput. Phys. Commun. 13690

[24] Lunt T. et al. 2012 Nucl. Fusion 52054013

[25] G.D.Conway et al. 2008 Proc. 22nd IAEA Fusion Eng. Conf. (Geneva) IAEA-CN-165/EX/P5-38

[26] Fitzpatrick R. 1993 Nucl. Fusion 331049

[27] De Bock M.F.M et al. 2008 Nucl. Fusion 48015007

[28] Waelbroeck F.L. 2009 Nucl. Fusion 49104025

[29] Yu Q. et al. 2009 Phys. Plasma 16042301

[30] Park G. et al. 2010 Phys. Plasma 17102503

[31] Liu Y. et al. 2010 Phys. Plasma 17122502

[32] Callen J.D. 2011 Nucl. Fusion 51094026

[33] Kaveeva E. and Rozhansky V. 2012 Nucl. Fusion 52054011

[34] Fitzpatrick R. 2012 Plasma Phys. Control. Fusion 54094002

[35] Coenen J.W. et al. 2011 Nucl. Fusion 51063030

[36] Conway G.D. et al. 2013 Proc. 40th EPS Conf. (Helsinki), ECA 37D P5.175

[37] Maraschek M. et al. 2013 Proc. 40th EPS Conf. (Helsinki), ECA 37D P4.127

[38] Fuchs J.C. et al. 2011 Proc. 38th EPS Conf. (Strasbourg) ECA 35G P1.090

[39] Fuchs J.C. et al. 2013 Proc. 40th EPS Conf. (Helsinki) ECA 37D P4.126

[40] Fischer R. et al. 2012 Plasma Phys. Control. Fusion 54115008

[41] Conway G.D. et al. 2008 Plasma Phys. Control. Fusion 50055009

[42] Simon P. 2014 Private communication

[43] Wahlberg C. 2009 Plasma Phys. Control. Fusion 51085006

[44] Huang Z. et al. 2013 Proc. 40th EPS Conf. (Helsinki), ECA 37D P2.175

[45] Fietz S. et al. 2013 Proc. 40th EPS Conf. (Helsinki), ECA 37D P5.158

[46] Robinson J.R. et al. 2012 Plasma Phys. Control. Fusion 54105007

[47] Xu Y. et al. 2010 Proc. 23rd IAEA Fusion Eng. Conf. (Daejon) EXC/9-3 